\title{
Discrete solitons in nonlinear Schrödinger lattices with a power-law nonlinearity
}

\author{
J. Cuevas
}

Grupo de Física No Lineal, Departamento de Física Aplicada I, Escuela Universitaria Politécnica, C/ Virgen de África, 7, 41011 Sevilla, Spain ${ }^{1}$

\author{
P.G. Kevrekidis
}

Department of Mathematics and Statistics, University of Massachusetts, Amherst MA 01003-4515

\section{D.J. Frantzeskakis}

Department of Physics, University of Athens, Panepistimiopolis, Zografos, Athens 15784, Greece

\section{B.A. Malomed}

Department of Physical Electronics, Faculty of Engineering, Tel Aviv University, Tel Aviv 69978, Israel

\begin{abstract}
We study the discrete nonlinear Schrödinger lattice model with the onsite nonlinearity of the general form, $|u|^{2 \sigma} u$. We systematically verify the conditions for the existence and stability of discrete solitons in the one-dimensional version of the model predicted by means of the variational approximation (VA), and demonstrate the following: monostability of fundamental solitons (FSs) in the case of the weak nonlinearity, $2 \sigma+1<3.68$; bistability, in a finite range of values of the soliton's power, for $3.68<2 \sigma+1<5$, and the presence of a threshold (minimum norm of the FS), for $2 \sigma+1 \geq 5$. We also perform systematic numerical simulations to study higher-order solitons in the same general model, i.e., bound states of the FSs. While all in-phase bound states are unstable, stability regions are identified for antisymmetric double solitons, and their triple counterparts. These numerical findings are supplemented by an analytical treatment of the stability problem, which allows quantitively accurate predictions for the stability features of such multipulses. When these waveforms are found to be unstable, we show, by means of direct simulations, that they self-trap into a persistent lattice breather, or relax into a stable FS, or sometimes decay completely.
\end{abstract}




\section{Introduction}

Discrete nonlinear Schrödinger (DNLS) equations represent a vast class of dynamical lattice models with many mathematical and physical applications [1]. The DNLS equation with the onsite cubic nonlinearity models, among others, an array of nonlinear-optical waveguides [2], that was originally implemented in an experiment as a set of parallel ribs made of a semiconductor material (AlGaAs) and mounted on a common substrate [3] (see also the review [4]). Quasi-discrete optical waveguide arrays can also be created as virtual photonic lattices in photorefractive crystals [5], the appropriate model being the DNLS with the saturable nonlinearity, known as the Vinetskii-Kukhtarev model, that was introduced more than 30 years ago [6], and has drawn a great deal of interest recently [7].

It was predicted [8] that the DNLS equation may also serve as a model for Bose-Einstein condensates (BECs) trapped in a strong optical lattice (a sinusoidal potential composed by the interference of laser beams), which was confirmed by the experiment [9] (see also the review [10]). In addition to the direct physical realizations in terms of nonlinear optics and BECs, the DNLS equations appear as universal asymptotic forms of various models based on chains of coupled oscillators. Accordingly, the solitons known in the DNLS equation represent intrinsic localized modes investigated in such chains theoretically [11] and experimentally [12].

Fundamental states supported by the DNLS equations are discrete solitons. In the DNLS equation with cubic and saturable nonlinearities, the solitons have been studied in detail (first of all, in one-dimensional models, but many results have been also obtained for two- and three-dimensional DNLS lattices) [1]. Equations with more complex nonlinearities are of interest too. In particular, applications to optics suggest the consideration of solitons in the DNLS equation with the onsite nonlinearity combining self-focusing cubic and self-defocusing quintic terms. Recently, localized states in this one-dimensional discrete model with the competing nonlinearities were studied in detail in Ref. [13], where it was shown that novel classes of solutions can be introduced by this competition.

On the other hand, a subject of general interest is also the study of various species of solitons and their stability in the DNLS equation with arbitrary onsite power nonlinearity, i.e.,

$$
i \dot{u}_{n}=-\epsilon\left(u_{n+1}+u_{n-1}-2 u_{n}\right) u_{n}-\left|u_{n}\right|^{2 \sigma} u_{n},
$$

where $u_{n}(t)$ are the lattice dynamical variables, the overdot stands for the

$\overline{1}$ Corresponding author. E-mail: jcuevas@us.es 
time derivative, $\epsilon>0$ is the lattice coupling constant, and $\sigma>0$ determines the nonlinearity power. This model was introduced in Ref. [14] (see also Ref. [15]), where, in particular, quasi-collapse was studied, in the case of $\sigma \geq 2$ (the critical or supercritical collapse [16] takes place in the continuum limit of the one-dimensional Eq. (1), i.e., the ordinary NLS equation, with $\sigma=2$ and $\sigma>2$, respectively). In particular, the case of $\sigma=2$, i.e., the quintic nonlinearity in Eq. (1), has also been argued to be of relevance in the case of BECs. In particular, in the limit of very tight transverse confinement, and for sufficiently low densities, an atomic BEC with repulsive interactions behaves like a one-dimensional gas of impenetrable bosons, the so-called Tonks-Girardeau gas [17]. In the framework of mean-field theory, it has been proposed that such a bosonic gas behaving like a system of free fermions, may be described by the continuum NLS equation with the defocusing (self-repulsive) quintic nonlinearity (without the cubic term) [18]. Then, similarly to the case of regular BECs, if this Tonks-Girardeau gas is trapped in a strong optical-lattice potential [19], it may be described by the DNLS Eq. (1) with $\sigma=2$ [strictly speaking, with the opposite sign in front of the nonlinear term; however, the equation may be cast in the form of Eq. (1) by means of the staggering transformation [1], i.e., $u_{n} \equiv(-1)^{n} e^{-4 i \epsilon t} \tilde{u}_{n}^{*}$.

Quite general predictions for solitons in the DNLS equation (1) were made, on the basis of the variational approximation (VA), in Ref. [20]:

(i) There is a critical value of the nonlinearity power, $\sigma_{\mathrm{cr}} \approx 1.42$, such that, for $\sigma<\sigma_{\text {cr }}$, exactly one soliton can be found for each value of the norm, $P \equiv \sum_{n=-\infty}^{+\infty}\left|u_{n}\right|^{2}$ (that is, there no threshold for the existence of the solitons, and no bistability).

(ii) In the range of $\sigma_{\mathrm{cr}}<\sigma<2$, three different solitons coexist in a certain finite interval of values of $P$, two stable and one unstable (It is worthwhile to note here that there is no such multistability in the continuum limit of the DNLS equation, where, in fact, solutions are analytically available for any $\sigma$ [21]). For values of the norm below and above this range, one can find a unique soliton, which is stable. In fact, this feature has been used in [22] to produce controllable switching (via the use of an internal mode) from one of these two stable stationary states to the other.

(iii) For $\sigma \geq 2$, there exists a threshold (minimum norm) necessary for the existence of solitons. For $\sigma>2$, there exist two solitons above the threshold, one narrow (stable), and one broad (unstable).

As concerns the predictions for the stability, they were made in Ref. [20] on the basis of the extrapolation of the Vakhitov-Kolokolov (VK) stability criterion [23], which is well known as a necessary condition for the stability of solitons in 
continuum NLS equations [16]. This generalization is natural for fundamental solutions, since the DNLS can be cast in the general Hamiltonian framework of [24], for which the VK criterion has been established as governing the stability of such solutions. The criterion states that, if a family of stationary soliton solutions can be found as

$$
u_{n}(t)=e^{i \Lambda t} v_{n}
$$

with real frequency $-\Lambda$ and real stationary function $v_{n}$, the soliton family may be stable in a region where $d P / d \Lambda>0$, and it is definitely unstable otherwise (the VK criterion is only a necessary one, as it ignores possible oscillatory instability of the solitons, that would be accounted for by complex instability eigenvalues, see below).

While the above predictions are quite important, they have never been subjected to consistent numerical verification, as far as we know. This is the first objective of the present work (the results essentially confirm the conclusions produced on by the VA and VK criterion, with a difference that a numerically accurate critical value of the power of the onsite nonlinearity is $\sigma_{\mathrm{cr}} \approx 1.34$, instead of the above-mentioned approximation, $\sigma_{\mathrm{cr}} \approx 1.42$ ). Following this line of the analysis, in Section 2, we recapitulate the VA for the fundamental (single-humped) solitons in Eq. (1), and in Section 3 systematic numerical results are reported, which verify the predictions of the VA.

Another objective of the work is to theoretically analyze and numerically construct higher-order (multi-humped) states, i.e., bound states of the fundamental solitons (FS). In the DNLS equation with the cubic nonlinearity, they were introduced in Ref. [25]. A general principle which predicts their stability was formulated in Ref. [26]: bound states of the FSs with opposite signs may be stable, while compounds built of in-phase fundamental solitons may be only unstable (see also Refs. [27], where multihumped complexes were studied in chain of coupled oscillators and the work of [28], where such states were examined in one and two dimensions for the cubic nonlinearity). In Section 4, we present a theoretical analysis for the stability of such multi-humped states, while in Section 5 we report numerical results for basic families of the bound states in the present system. In agreement with the aforementioned principle, the bound states of two or three in-phase FSs are found to be always unstable (therefore, they are not considered in detail), while complexes of out-of-phase two or three FSs may be stable.

In Sections 3 and 5, we also present typical examples of direct simulations that illustrate the development of instability of fundamental and bound-state solitons, in cases when they are unstable. We find that there are three potential outcomes of these "numerical experiments": the waveforms may transform themselves into persistent discrete breathers, or relax into stable FSs, or completely decay into linear waves. 


\section{The variational approximation for fundamental solitons}

Substituting the general expression (2) for the stationary solitons in Eq. (1), one arrives at the following equation for the real discrete waveform $v_{n}$,

$$
\Lambda v_{n}=\epsilon\left(v_{n+1}+v_{n-1}-2 v_{n}\right)+v_{n}^{2 \sigma+1} .
$$

Equation (3) can be derived from the Lagrangian

$$
L=\sum_{n=-\infty}^{+\infty}\left[\epsilon\left(v_{n+1}+v_{n-1}\right) v_{n}-(\Lambda+2 \epsilon) v_{n}^{2}+\frac{1}{\sigma+1} v_{n}^{2(\sigma+1)}\right] .
$$

The VA for fundamental discrete solutions, elaborated in Ref. [20] (see also Ref. [13]) was based on the simple exponential ansatz,

$$
v_{n}=A e^{-a|n|},
$$

with variational parameters $A$ and $a$ (which, obviously, determine the amplitude and inverse size of the soliton). Then, substituting the ansatz in the Lagrangian, one can perform the summation explicitly, which yields the effective Lagrangian,

$$
L_{\mathrm{eff}}=2 \epsilon P \operatorname{sech} a-(\Lambda+2 \epsilon) P+\frac{P^{\sigma+1}}{\sigma+1} \frac{\operatorname{coth}((\sigma+1) a)}{\operatorname{coth}^{\sigma+1} a} .
$$

The norm of the ansatz (5), which appears in Eq. (6), is given by

$$
P \equiv \sum_{n=-\infty}^{+\infty} v_{n}^{2}=A^{2} \operatorname{coth} a
$$

The Lagrangian (6) gives rise to the variational equations, $\partial L_{\text {eff }} / \partial P=\partial L_{\text {eff }} / \partial a=$ 0 , which constitute the basis of the VA [29]. They predict relations between the norm, frequency, and width of the FSs within the framework of the VA, namely

$$
\begin{aligned}
P^{\sigma} & =\frac{4 \epsilon \cosh ^{\sigma} a \sinh ^{2}(\sigma+1) a}{\sinh ^{\sigma-1} a(\sinh 2(\sigma+1) a-\sinh 2 a)}, \\
\Lambda & =2 \epsilon(\operatorname{sech} a-1)+P^{\sigma} \frac{\operatorname{coth}(\sigma+1) a}{\operatorname{coth}^{\sigma+1} a}
\end{aligned}
$$

These analytical predictions, implicitly relating $P$ and $\Lambda$ through their parametric dependence on $a$, will be compared with numerical findings below.

\section{Numerical results: fundamental solitons}

Using the scaling invariance of Eq. (3), we will present numerical findings for $\Lambda \equiv 1$ (in most cases), using $\epsilon$ and $\sigma$ as free parameters. We display numerically 
obtained results for FSs in Eq. (1) with four distinct values of the nonlinearity power, viz., $\sigma=1,1.5,2$, 3. As explained in Introduction, $\sigma=1$ and 2, corresponding to the cubic and quintic nonlinearity, respectively, are relevant to applications in nonlinear optics and BECs.

Localized real solutions $v_{n}$ to Eq. (3) were found starting from the anticontinuum limit, $\epsilon=0$, where they can be constructed trivially, and continuing them up to a required value of $\epsilon$. After that, the stability of the solitons was analyzed by taking a perturbed solution in the form

$$
u_{n}(t)=e^{i \Lambda t}\left[v_{n}+a_{n} \exp (-\lambda t)+b_{n}^{*}\left(\lambda^{*} t\right)\right],
$$

and linearizing Eqs. (1) with respect to small perturbation modes $a_{n}$ and $b_{n}$. This leads to an eigenvalue problem (for the instability growth rate $\lambda$, that may be complex), based on the following coupled linear equations,

$$
\begin{aligned}
& i \lambda a_{n}=-\epsilon \Delta_{2} a_{n}+\Lambda a_{n}-(\sigma+1) v_{n}^{2 \sigma} a_{n}-\sigma v_{n}^{2 \sigma} b_{n}, \\
& i \lambda b_{n}=\epsilon \Delta_{2} b_{n}-\Lambda b_{n}+(\sigma+1) v_{n}^{2 \sigma} b_{n}+\sigma v_{n}^{2 \sigma} a_{n} .
\end{aligned}
$$

This problem was solved by a standard numerical eigenvalue solver.

First, typical examples of numerically found FSs, as well as double and triple bound states of in-phase and out-of-phase (sign-changing) types, are displayed in Fig. 1. The FSs exist at all values of $\epsilon$ for all $\Lambda$. In the cubic model, with $\sigma=1$, they are stable for all $\epsilon$, which is a well-known fact [1]. However, in the model with higher nonlinearities, $\sigma=2$ (quintic) and $\sigma=3$, they are stable only in finite intervals, $0<\epsilon<1.079$ and $0<\epsilon<0.679$, respectively. For the quartic nonlinearity, with $\sigma=1.5$, the FS solutions are stable in two intervals: a finite one, $0<\epsilon<1.482$, and a semi-infinite region, $\epsilon>3.437$. These results are summarized in Fig. 2, which clearly demonstrates that shapes of the FS family, if represented in the form of the $P(\epsilon)$ dependence, are nearly the same for all the aforementioned values of $\sigma$, while their stability properties are drastically different.

Stability changes are closely related to the above-mentioned VK criterion, $d P / d \Lambda>0$. Figure 3 shows $P$ as a function of $\epsilon$ for fixed $\Lambda \equiv 1$, therefore the VK criterion cannot be directly applied to it. However, rescaling $v_{n} \equiv$ $(2 \epsilon)^{1 / 2(\sigma+1)} \tilde{v}_{n}$, i.e., $P \equiv(2 \epsilon)^{1 /(\sigma+1)} \tilde{P}$, one can cast the stationary equation (3) with $\Lambda=1$ and arbitrary $\epsilon$ in a different form, with $\epsilon=1 / 2$ and $\Lambda=1 /(2 \epsilon)$, which yields plots $P(\Lambda)$ shown in Fig. 3, together with the same dependencies predicted by the VA as per Eqs. (8) and (9). The comparison with results of the computation of the stability eigenvalues from Eqs. (11) demonstrates that the stability change takes place exactly at points $d P / d \Lambda=0$, as expected (per our arguments above), in full agreement with the VK criterion. The two sign 
(a)

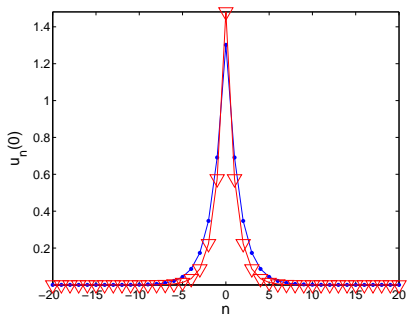

(d)

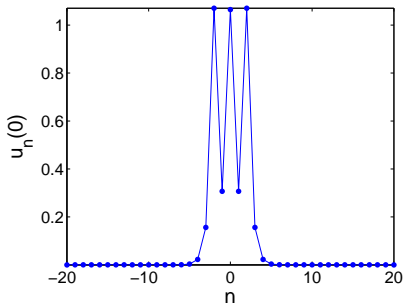

(b)

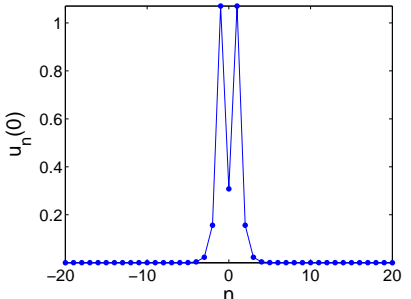

(e)

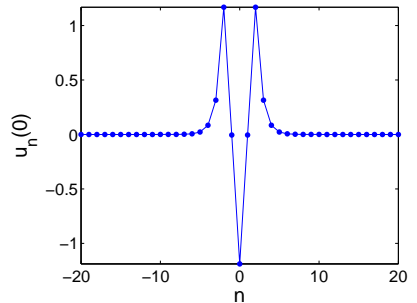

(c)

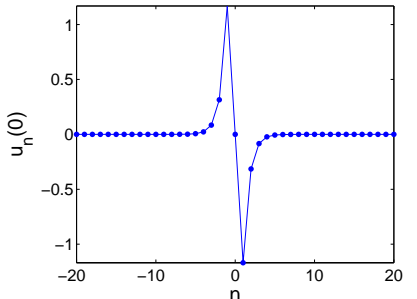

Fig. 1. Typical profiles of fundamental solitons and bound states in the DNLS equation with the quintic nonlinearity, $\sigma=2$. A fundamental pulse is shown in panel (a) for $\epsilon=1$; Triangles represent the profile predicted by the VA. Double pulses: in-phase ones, structured like $(1,0,1)$, for $\epsilon=0.2(\mathrm{~b})$, and out-of-phase double pulses, of the type $(1,0,-1)$, for $\epsilon=0.5$ (c). Triple pulses: of types $(1,0,1,0,1)$ for $\epsilon=0.2$ (d), and $(1,0,-1,0,1)$ for $\epsilon=0.5$ (e). In all figures, except for Fig. 3, the solitons and their families are shown for the intrinsic frequency fixed by the scaling, $\Lambda \equiv 1$.

changes of $d P / d \Lambda$ also explain the bistability observed (in Fig. 2) at $\sigma=1.5$.

As shown in Ref. [20], the VA predicts that all FSs are stable for $\sigma<\sigma_{\mathrm{cr}} \approx$ 1.42 , with a single FS corresponding to each value of $P$, and that bistability arises in a finite interval of values of the norm in the FS family, for $1.42<\sigma<$ 2. Further, for $\sigma \geq 2$, there is a threshold (minimum) value of the norm, $P_{\mathrm{thr}}$, necessary for the existence of the solitons. For $\sigma>2$, there are two FS families with $P>P_{\mathrm{thr}}$, one stable and one unstable. The numerical results confirm all these conclusions, with a difference that the numerically found value of $\sigma_{\mathrm{cr}}$ is lower than the aforementioned one predicted by the VA, viz., $\sigma_{\mathrm{cr}}=1.34$ (rather than $\sigma_{\mathrm{cr}}=1.42$ ).

Getting back to the normalization with $\Lambda \equiv 1$ and varying $\epsilon$, we notice that the predictions of the VA can be reformulated as follows. At $\sigma<\sigma_{\mathrm{cr}}$, the FSs are stable for all $\epsilon$. In the case of $\sigma_{\mathrm{cr}}<\sigma<2$, the solitons are stable in intervals $0<\epsilon<\epsilon_{1}(\sigma)$ and $\epsilon>\epsilon_{2}(\sigma)$, being unstable in between, at $\epsilon_{1}(\sigma)<\epsilon<\epsilon_{2}(\sigma)$. Here, $\epsilon_{1}(\sigma)$ and $\epsilon_{2}(\sigma)$ represent the stability borders shown in the left and right panels of Fig. 4. Both the destabilization of the FS at $\epsilon=\epsilon_{1}(\sigma)$ and its restabilization at $\epsilon=\epsilon_{2}(\sigma)$ are accounted for by the sign change in the 

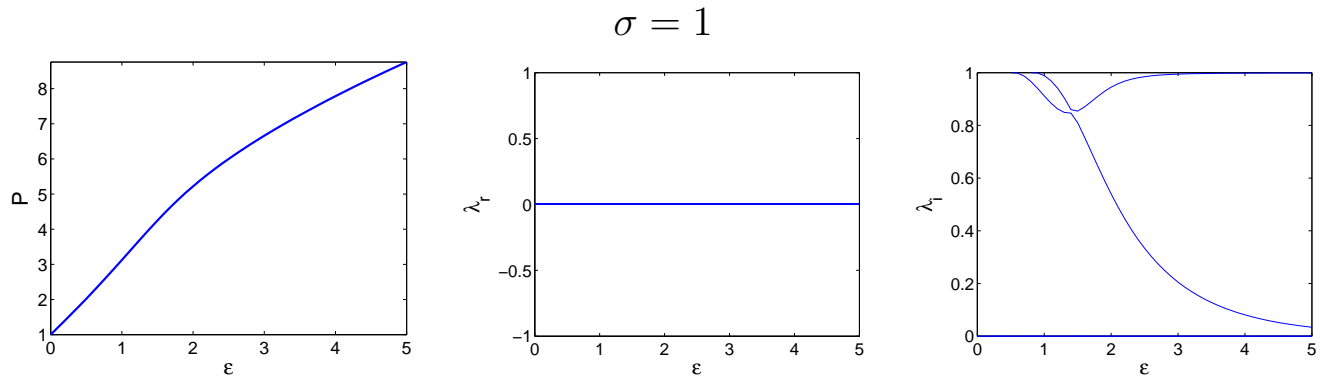

$\sigma=1.5$
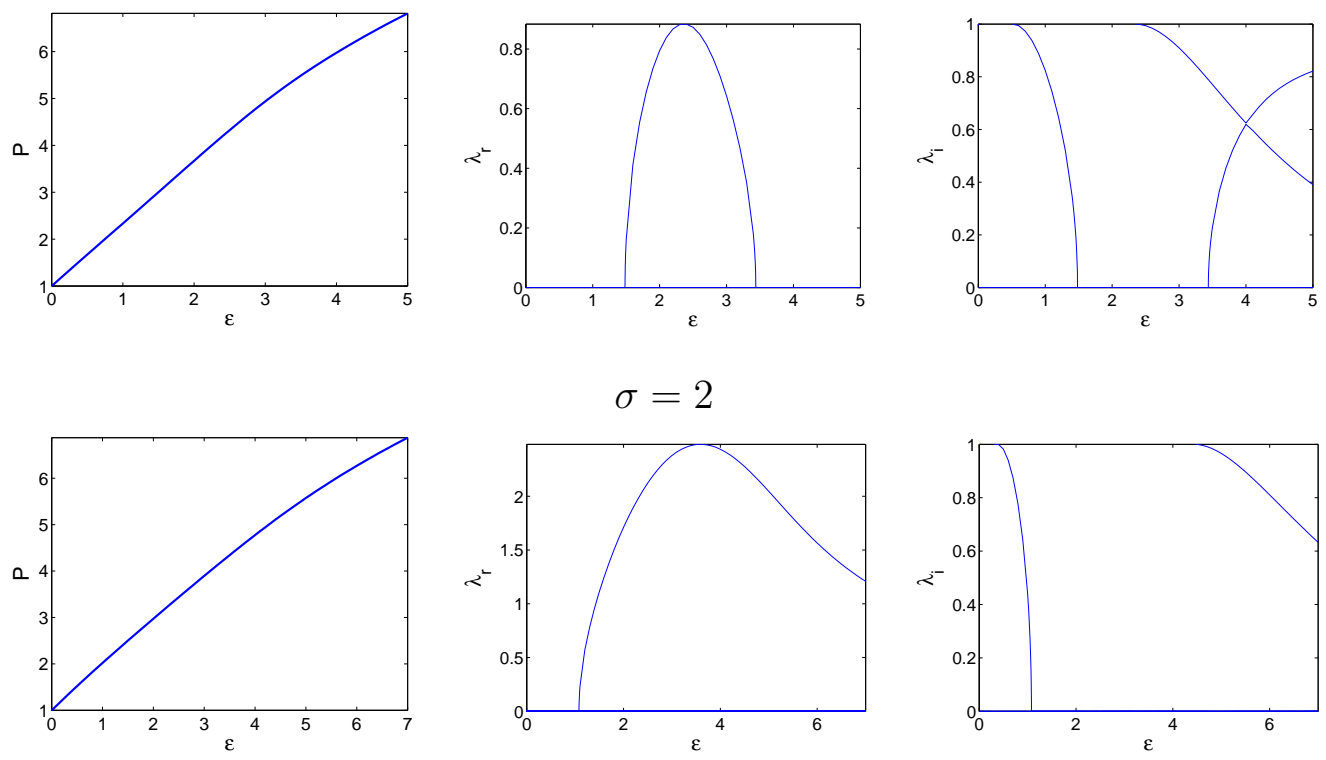

$$
\sigma=3
$$
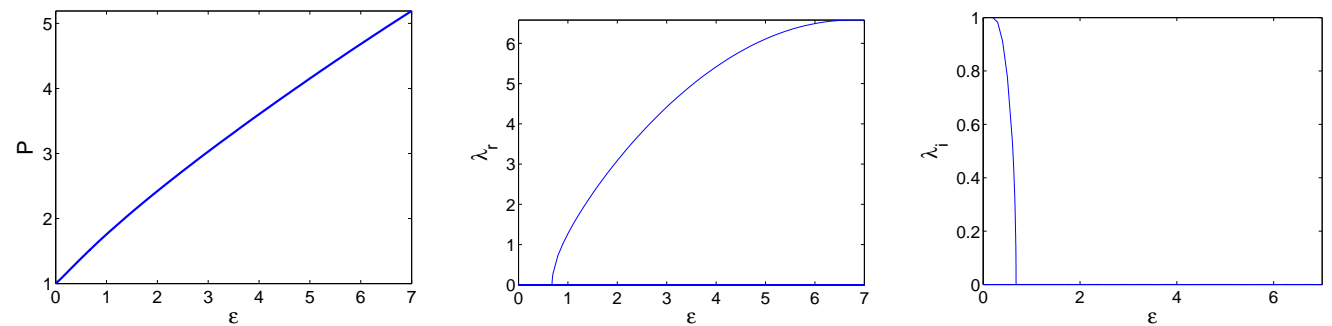

Fig. 2. Families of FS (fundamental-soliton) solutions. Left panels: the FS norm versus the coupling constant $\epsilon$. The center and right panels show, respectively, the real and imaginary parts of the linear stability eigenvalues $\lambda \equiv \lambda_{r}+i \lambda_{i}$, see Eq. (10), versus $\epsilon$.

VK criterion, hence these bifurcations are related to the exponential (nonoscillatory) instabilities, which occur via real eigenvalues $\lambda$. These eigenvalues, as can be seen in the right and middle panels of Fig. 2, bifurcate from the lower edge of the phonon band at $\lambda_{i}=\Lambda$ at some nonzero value of $\epsilon$ and subsequently move towards $\lambda=0$. In subcritical cases, they only arrive there 
(a)

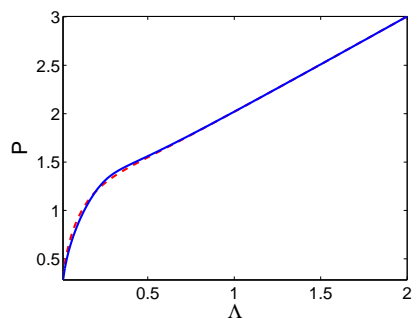

(c)

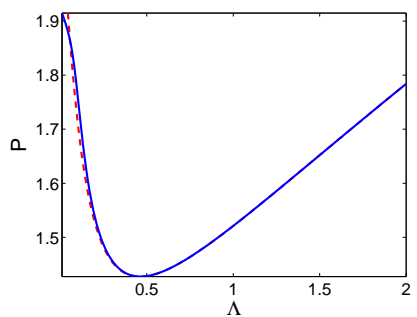

(b)

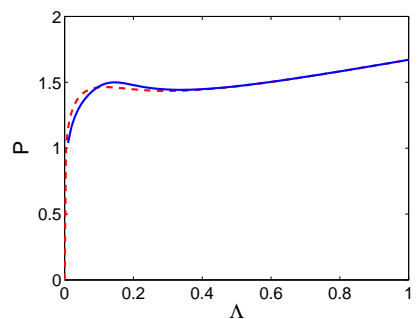

(d)

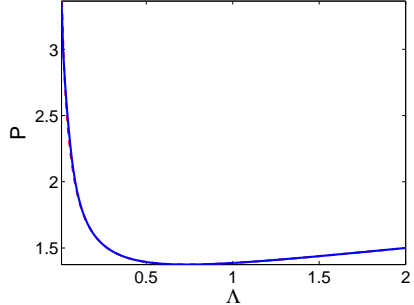

Fig. 3. The norm of the fundamental-soliton family versus $\Lambda$ for (a) $\sigma=1$, (b) $\sigma=1.5$, (c) $\sigma=2$, (d) $\sigma=3$. Full lines display numerical results, while the dashed curves correspond to the predictions of the variational approximation.

as $\epsilon \rightarrow \infty$; for cases with $\sigma_{c r}<\sigma<2$, they cross it twice, leading to the bistability feature, while for $\sigma \geq 2$, they only cross it once. Note that $\epsilon \rightarrow \infty$ corresponds to the continuum limit, which explains why the restabilization occurs only for $\sigma<2$, and is impossible for $\sigma \geq 2$ (formally speaking, this means $\left.\epsilon_{2}(\sigma=2)=\infty\right)$ : the continuum one-dimensional NLS equations with the nonlinearity corresponding to $\sigma \geq 2$ gives rise to collapse [16], hence the solitons in this continuum equation are unstable. Figure 4 demonstrates that the VA predicts both bifurcations, at $\epsilon=\epsilon_{1,2}(\sigma)$, in a qualitatively correct form, although it is less accurate quantitatively in the vicinity of the transition points $\sigma=\sigma_{c r}$ and $\sigma=2$. One expects that, in the neighborhood of these, the detailed structure of the soliton may be relevant and hence its simple exponential representation by the VA may not be sufficient for quantitative purposes.

To complete the consideration of the fundamental solitons, we have simulated the evolution of those among them which are predicted to be unstable by the VK criterion and computation of the stability eigenvalues (see Eqs. (11)). As shown in the upper part of Fig. 5, the milder instability (i.e., with smaller growth rate as represented by the largest eigenvalue real part) of the soliton in the window $\epsilon_{1}<\epsilon<\epsilon_{2}$, which is possible in the case of $\sigma_{\mathrm{cr}}=1.34<\sigma<$ 2 , transforms it into a persistent lattice breather, as mentioned in Ref. [14] (although the original instability is not oscillatory, see above). On the other hand, the stronger instability of the soliton in the examined case for $\epsilon>\epsilon_{2}$, 

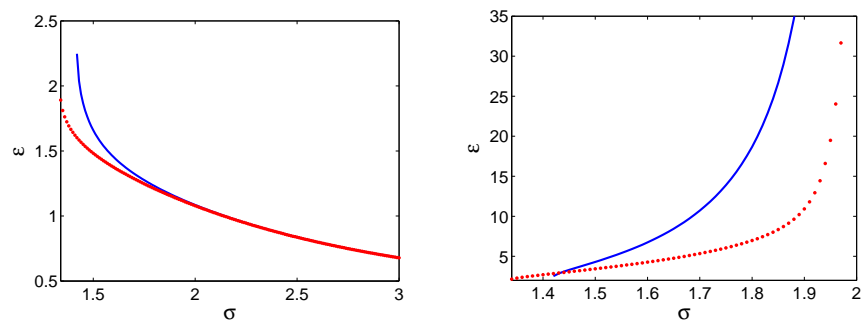

Fig. 4. Locations of the two bifurcations that account for the exponential (non-oscillatory) destabilization and subsequent restabilization (the left and right panels, respectively) of the fundamental solitons (subject to the normalization $\Lambda \equiv 1$ ) in the plane of $(\sigma, \epsilon)$. The (blue) line and (red) dots represent predictions of the variational approximation and numerical results, respectively. The restabilization corresponding to the right panel does not occur for $\sigma \geq 2$.
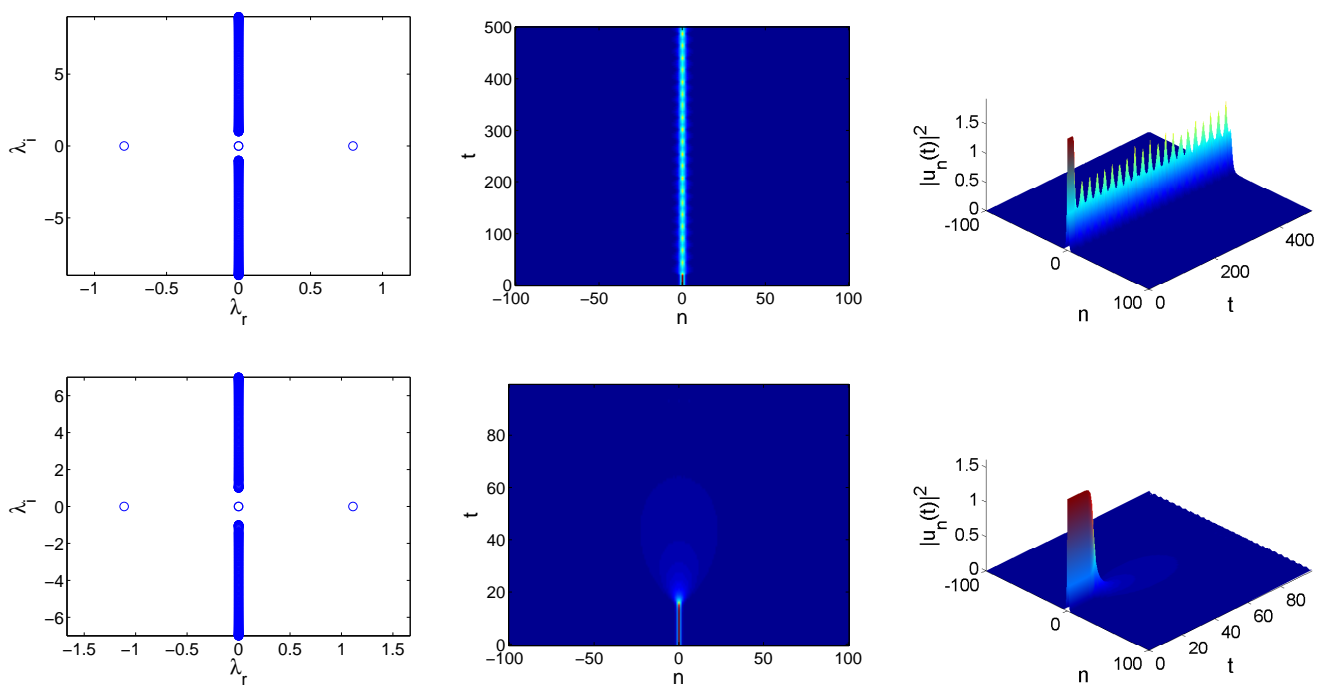

Fig. 5. Typical examples of weakly and strongly unstable fundamental solitons, corresponding, respectively, to $\sigma=1.5, \epsilon=2$ (top) and $\sigma=2, \epsilon=1.5$ (bottom). The left panels show the spectral planes of the instability eigenvalues for small perturbations around the soliton. The temporal development of the instabilities is shown in the central and right panels, by means of density contours and three-dimensional plots. The weak instability turns the soliton into a persistent breather, while the strong instability destroys it. In the direct simulations, perturbations are generated solely by numerical truncation errors.

at $\sigma \geq 2$ at the bottom panel of the figure 5 , tends to completely destroy the soliton. 


\section{Analytical results: multisoliton bound states}

We now turn to multisoliton bound states, adapting the analytical techniques of [30] to the present problem. In particular, starting from the Eqs. (11), we use the decomposition $a_{n}=z_{n}+i w_{n}, b_{n}=z_{n}-i w_{n}$, to rewrite the eigenvalue problem as

$$
\begin{aligned}
-\lambda w_{n} & =L_{+} z_{n}=-\epsilon \Delta_{2} z_{n}+\left(1-(2 \sigma+1) v_{n}^{2 \sigma}\right) z_{n} \\
\lambda z_{n} & =L_{-} w_{n}=-\epsilon \Delta_{2} w_{n}+\left(1-v_{n}^{2 \sigma}\right) w_{n} .
\end{aligned}
$$

Combining these equations one obtains $\lambda^{2} w_{n}=-L_{+} L_{-} w_{n}$. Near the anticontinuum limit of $\epsilon=0$, for each of the excited sites (with $\left|v_{n}\right|=1$ ), the action of the $L_{+}$simplifies into a multiplicative operation, which allows us to invert the operator and accordingly obtain the equation for the eigenvalues

$$
\lambda^{2}=-\frac{\left(\mathbf{w}, L_{-} \mathbf{w}\right)}{\left(\mathbf{w}, L_{+}^{-1} \mathbf{w}\right)}
$$

where $\mathbf{w}$ is the vector with elements $w_{n}$. Then, using the fact that $\lim _{\epsilon \rightarrow 0}\left(\mathbf{w}, L_{+}^{-1} \mathbf{w}\right)=$ $-(2 \sigma)^{-1}$, we obtain the following expression for the eigenvalues

$$
\lambda^{2}=\sqrt{2 \sigma}\left(\mathbf{w}, L_{-} \mathbf{w}\right)
$$

as the appropriate generalization of Eq. (3.11) of [30]. However, following the theory of [30], the eigenvalues of $L_{-}$can be evaluated as $\epsilon^{s} \gamma$. In the above expression, $s$ is the distance between the sites of the configuration (e.g., for nearest-neighbors $s=1$, while for next-nearest-neighbors $s=2$, for the configurations considered herein), while $\gamma$ 's are the eigenvalues of the $L \times L$ matrix (where $L$ is the number of sites excited in the anti-continuum limit) with off-diagonal entries: $M_{n, n+1}=M_{n+1, n}=-\cos \left(\theta_{n+1}-\theta_{n}\right)$ and diagonal entries $M_{n, n}=\left(\cos \left(\theta_{n-1}-\theta_{n}\right)+\cos \left(\theta_{n+1}-\theta_{n}\right)\right)$. In the case of $L=2$ excited sites, it is straightforward to obtain that $\gamma=0$ or $\gamma=2 \cos \left(\theta_{1}-\theta_{2}\right)$, where $\theta_{1,2}$ are the respective phases of the sites in the limit of $\epsilon=0$. On the other hand, for $L=3$ excited sites, there is a $\gamma=0$ eigenvalue, in addition to two non-vanishing ones

$$
\begin{aligned}
\gamma_{1} & =\cos \left(\theta_{2}-\theta_{1}\right)+\cos \left(\theta_{3}-\theta_{2}\right) \\
& \pm \sqrt{\cos ^{2}\left(\theta_{2}-\theta_{1}\right)-\cos \left(\theta_{2}-\theta_{1}\right) \cos \left(\theta_{3}-\theta_{2}\right)+\cos ^{2}\left(\theta_{3}-\theta_{2}\right)} .
\end{aligned}
$$

Similarly to [30], one can observe that the configurations that will be stable are the ones where adjacent excited sites are out of phase with each other. 

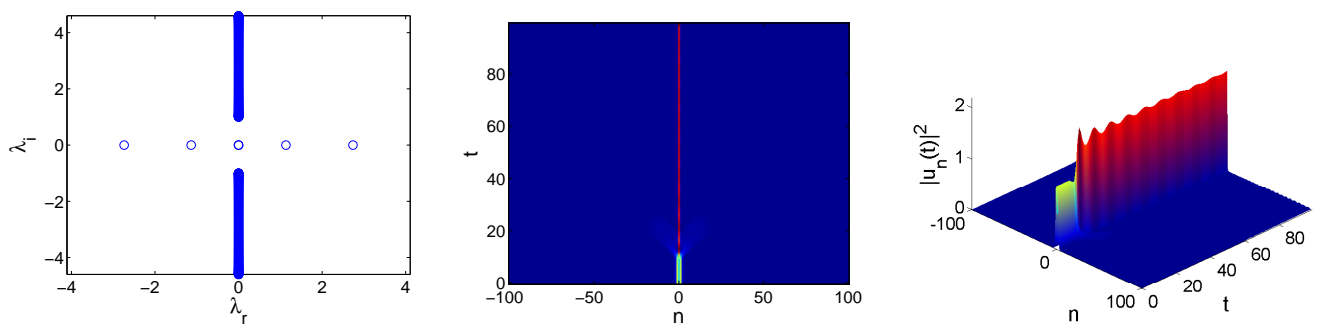

Fig. 6. The same as in Fig. 5 for a moderately unstable symmetric double pulse of type $(+1,0,+1)$, with $\sigma=3$ and $\epsilon=1$. The instability transforms it into a fundamental soliton with residual intrinsic oscillations.

Focusing, in particular, on the examples that will be presented in detail in the numerical section below, we have that for the configuration $(+1,0,-1)$, the above calculation predicts that the small (near zero) eigenvalues will be $\lambda=0$ and $\lambda= \pm i 2 \sqrt{\sigma} \epsilon$. On the other hand, for the case of $(+1,0,-1,0,+1)$ also examined below, our theoretical prediction is that the three relevant small eigenvalue pairs should be $\lambda=0, \lambda= \pm i \sqrt{2 \sigma} \epsilon$ and $\lambda= \pm i \sqrt{6 \sigma} \epsilon$. We now turn to a comparison of the full numerical results with these analytical predictions.

\section{Numerical results: multisoliton bound states}

\subsection{Two-humped states}

Figure 1 shows various types of two- and three-humped stationary solutions, that may be regarded as in-phase and out-of phase bound states of the FSs. In accordance with the general prediction of Ref. [26], the in-phase bound states, of types $(+1,0,+1)$ and $(+1,0,+1,0,+1)$ (panels $(b)$ and $(d)$ in Fig. 1) are unstable for all nonzero values of the lattice coupling, $\epsilon>0$. Direct simulations, displayed in Fig. 6, demonstrate that the unstable two-humped states may relax, through oscillatory evolution, into a stable FS. Similar results are demonstrated by direct simulations of the evolution of unstable threehumped states of type $(+1,0+1,0,+1)$ (not shown here).

On the other hand, antisymmetric bound states of type $(+1,0,-1)$, see Fig. $1(\mathrm{c})$, may be stable, in consonance with our theoretical predictions above. Existence and stability conditions for the antisymmetric double solitons are summarized in Fig. 7. They were found to exist below a maximum value of $\epsilon$, which is $\epsilon_{\max }=1.086,1.789,2.584$ and 4.426, for $\sigma=1,1.5,2$, and $\sigma=3$, respectively (the presence of the upper limit for their existence is natural, as they, obviously, have no counterparts in the continuum limit, which corresponds to $\epsilon \rightarrow \infty)$. For more details on the nature and bifurcation structure 
of the termination of such branches, the interested reader is directed to the detailed study of [31]. On the other hand, these branches may become unstable for $\epsilon>\epsilon_{\mathrm{cr}}^{(1)}$, where the critical values are $\epsilon_{\mathrm{cr}}^{(1)}=0.431,0.331,0.264$ and 0.192 , for $\sigma=1, \sigma=1.5, \sigma=2$ and $\sigma=3$, respectively. Beyond this critical point, the antisymmetric double soliton undergoes destabilization via a Hamiltonian Hopf bifurcation, originating from the collision of two imaginary eigenvalues with opposite Krein signature [24,30]. One of these two eigenvalues is the small eigenvalue, bifurcating from $\lambda=0$ for $\epsilon \neq 0$, according to our theoretical predictions of the previous section. Notice the level of agreement between the theoretical prediction and the numerical results for these eigenvalues in the right panels of Fig. 7. The coupling strength for which this eigenvalue $\pm 2 \epsilon \sqrt{\sigma} i$ reaches the bottom of the phonon band $\pm i(0.5,0.4082$, 0.3536 and 0.2887 for $\sigma=1,1.5,2,3$ respectively) yields a fair upper bound for the relevant critical point, since the collision occurs with an eigenvalue bifurcating from the phonon band edge. This scenario is fundamentally different from the above-described situation for the FSs, which could be destabilized only by non-oscillatory instabilities, associated with real eigenvalues. For values of $\epsilon>\epsilon_{\mathrm{cr}}^{(1)}$, the antisymmetric double solitons may become more unstable due to a real eigenvalue pair emerging through a mechanism similar to the one discussed in the case of the FSs, beyond a second critical value, $\epsilon_{\mathrm{cr}}^{(2)}=1.002$, 1.283, 1.251, and 0.961 for $\sigma=1, \sigma=1.5, \sigma=2$ and $\sigma=3$, respectively.

If an antisymmetric double soliton is unstable, direct simulations demonstrate either spontaneous transformation into a FS, or complete decay. Typical examples of the instability development are presented in Fig. 8.

\subsection{Three-humped states}

It was mentioned above that in-phase bound states of three FSs, like the one displayed in Fig. 1(d), are unstable. On the other hand, sign-changing triple bound states, of the type $(+1,0,-1,0,+1)$, an example of which is displayed in Fig. 1(e), may be stable. Similarly to other bound states, solitons belonging to this species feature an upper existence limit, $\epsilon<\epsilon_{\max }=1.066,1.758,2.544$, and 4.369 for $\sigma=1, \sigma=1.5, \sigma=2$ and $\sigma=3$, respectively. Like in the case of antisymmetric double solitons considered above, the stability region of the triple states is bounded by the condition $\epsilon \leq \epsilon_{\mathrm{cr}}^{(1)}=0.375,0.284,0.227$, and 0.165 for $\sigma=1, \sigma=1.5, \sigma=2$ and $\sigma=3$, respectively. At $\epsilon-\epsilon_{\mathrm{cr}}^{(1)} \rightarrow 0$, the triple solitons are destabilized through a Hamiltonian Hopf bifurcation. This occurs between the largest one of the two small pairs of eigenvalues of this case (theoretically predicted as $\pm \sqrt{6 \sigma} \epsilon i$ and $\pm \sqrt{2 \sigma} \epsilon i$ ) and the lower edge (or an eigenvalue bifurcating from the lower edge) of the phonon band. Once again, the theoretical prediction for the collision of the largest eigenvalue with the phonon band edge, being $\epsilon=0.4082,0.3333,0.2887$ and 0.2357 yields a 
$\sigma=1$
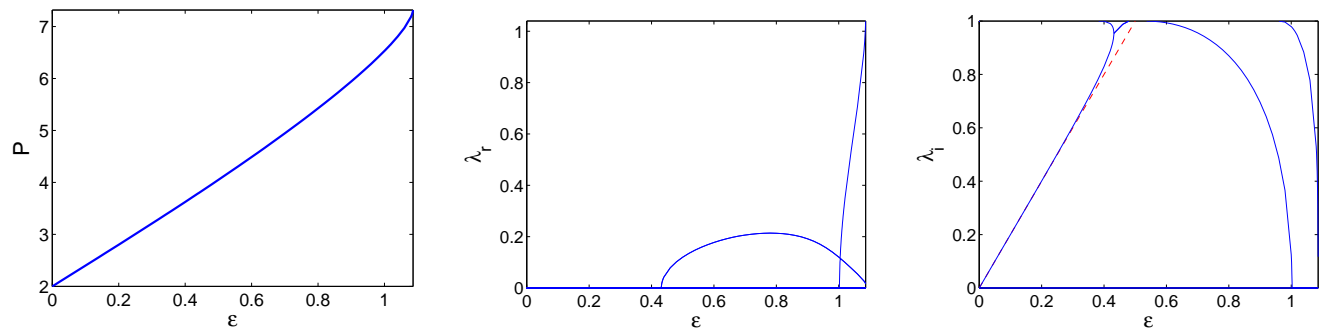

$\sigma=1.5$
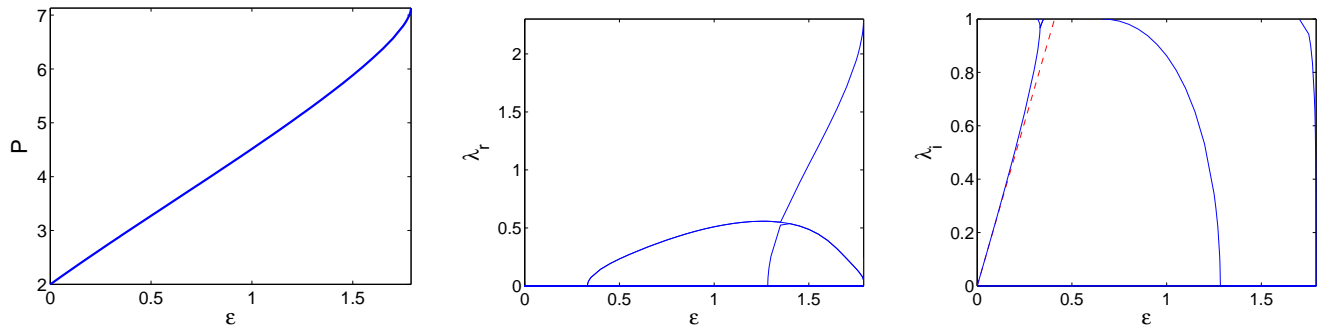

$$
\sigma=2
$$
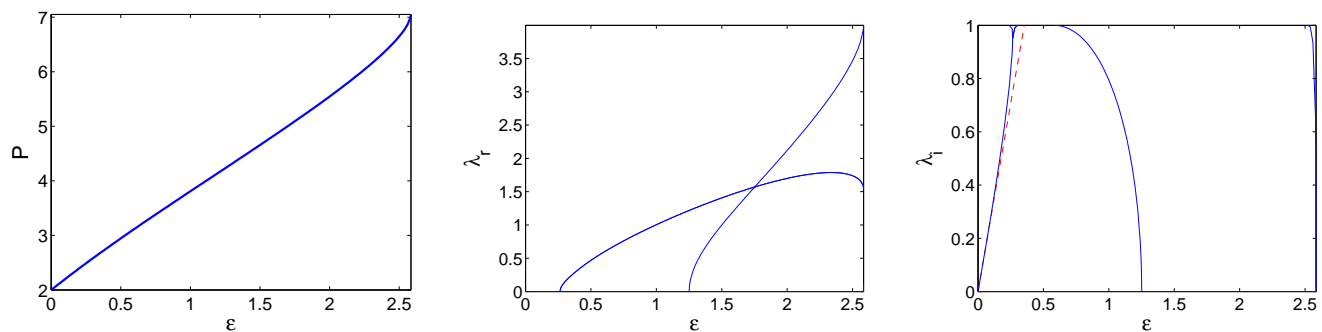

$$
\sigma=3
$$
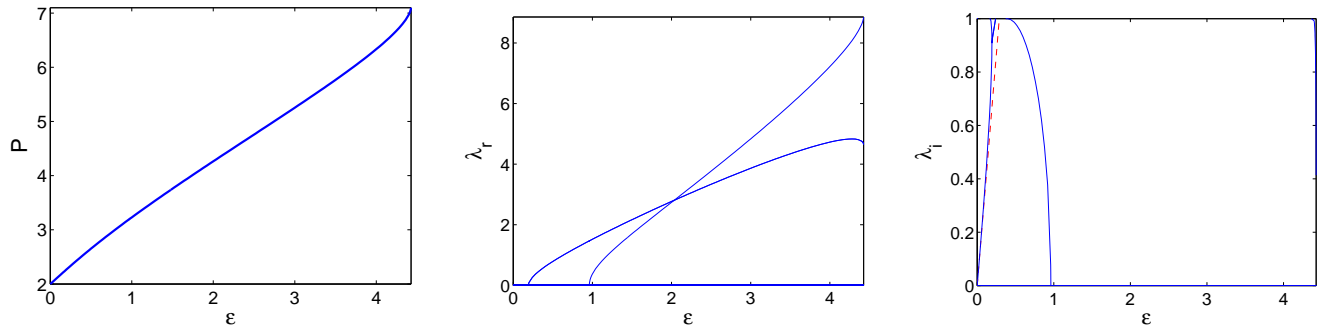

Fig. 7. The same as Fig. 2, but for the family of antisymmetric double solitons of type $(+1,0,-1)$.

reasonable upper bound for the location of the relevant destabilization point.

Naturally, an additional Hopf bifurcation occurs at $\epsilon=\epsilon_{\mathrm{cr}}^{(2)}=0.548,0.447$, 0.363 , and 0.266 for $\sigma=1,1.5,2$, and $\sigma=3$, respectively, due to the collision of the second small pair of eigenvalues with an eigenvalue pair bifurcating from the phonon band. Finally, in addition to these two Hamiltonian Hopf 

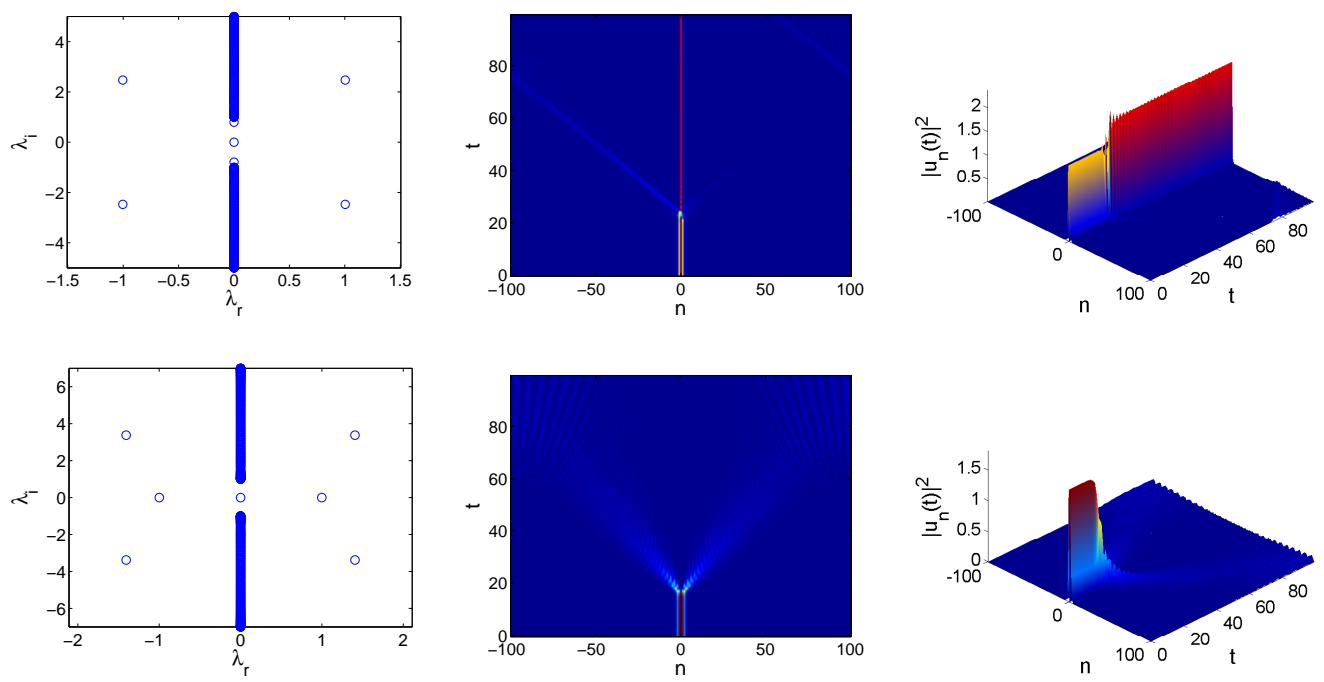

Fig. 8. The same as in Figs. 5 and 6, but for unstable double antisymmetric solitons of type $(+1,0,-1)$, with $\sigma=3$ and $\epsilon=1$ (top) and $\epsilon=1.5$ (bottom).

bifurcations, resulting in two quartets of eigenvalues, there is also an exponential instability, associated with the mechanism of destabilization present in the case of FSs which occurs for $\epsilon=\epsilon_{\mathrm{cr}}^{(3)}=1.066, \epsilon=1.758, \epsilon=1.489$ and $\epsilon=1.224$ for $\sigma=1, \sigma=1.5, \sigma=2$ and $\sigma=3$, respectively. All of these findings concerning the existence and stability of the triple solitons are summarized in Fig. 9.

Typical examples of the evolution of unstable three-humped states are displayed in Fig. 10. It is seen that they either relax into stable FS, which may be accompanied by emission of radiation jets, or they may completely decay.

\section{Conclusion}

Concluding, we have revisited the discrete-NLS model in one dimension, with the onsite nonlinearity of arbitrary power. One objective was to check the validity of the predictions of the VA (variational approximation), reported in Ref. [20] more than ten years ago, against systematic numerical results. We have demonstrated that the qualitative predictions of the VA are correct: monostability of the FSs (fundamental solitons) in the case of the weak nonlinearity (with power $2 \sigma+1<3.68$, which includes the cubic nonlinearity), bistability, in a certain interval of values of the soliton's power, for $3.68<2 \sigma+1<5$, and the presence of a threshold (minimum value of the norm), necessary for the existence of the solitons, for $2 \sigma+1 \geq 5$, i.e., for quintic and stronger nonlinearities. The only essential correction to the VA results is that the bor- 
$\sigma=1$
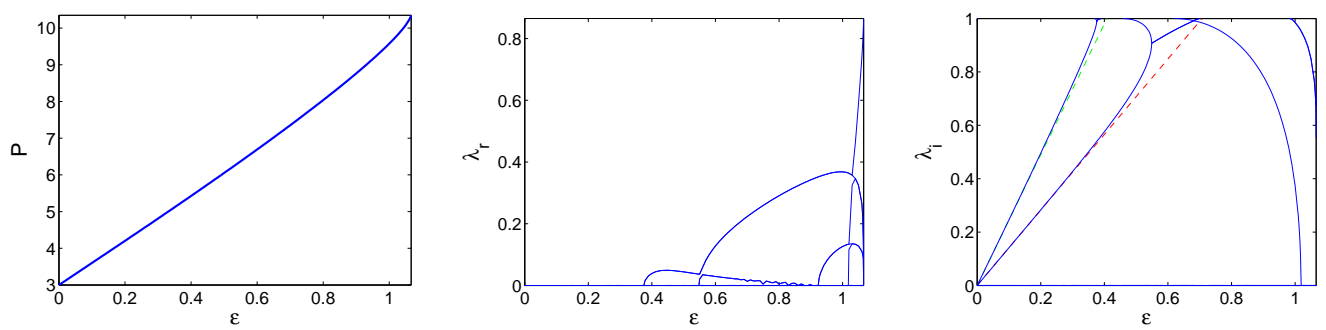

$\sigma=1.5$
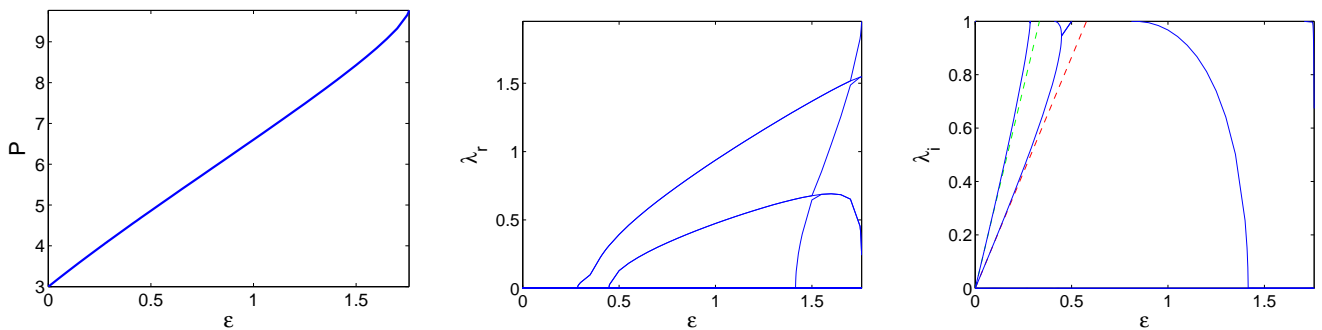

$$
\sigma=2
$$
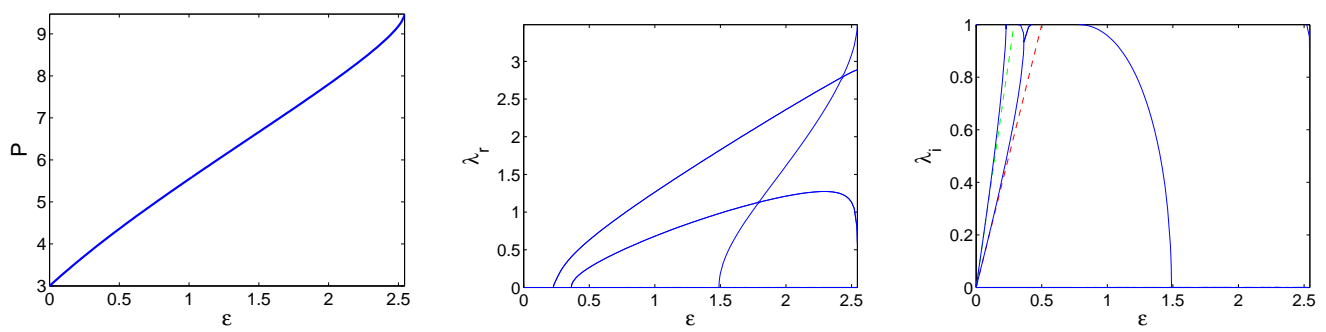

$$
\sigma=3
$$
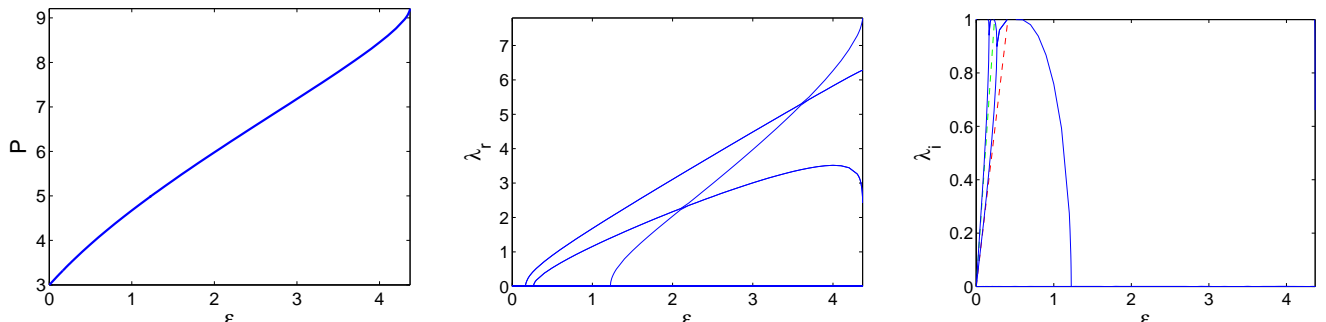

Fig. 9. Same as Figs. 2 and 7 , but for the family of triple solitons of type $(+1,0,-1,0,+1)$.

der between the weak and moderate nonlinearity was predicted by the VA at $2 \sigma+1 \approx 3.84$, which slightly differs the aforementioned numerically exact value, 3.68 .

The second part of the present work was dealing with bound states of FSs in the same class of models. Both through analytical considerations and through numerical results, it was concluded that, as predicted earlier, all in-phase 

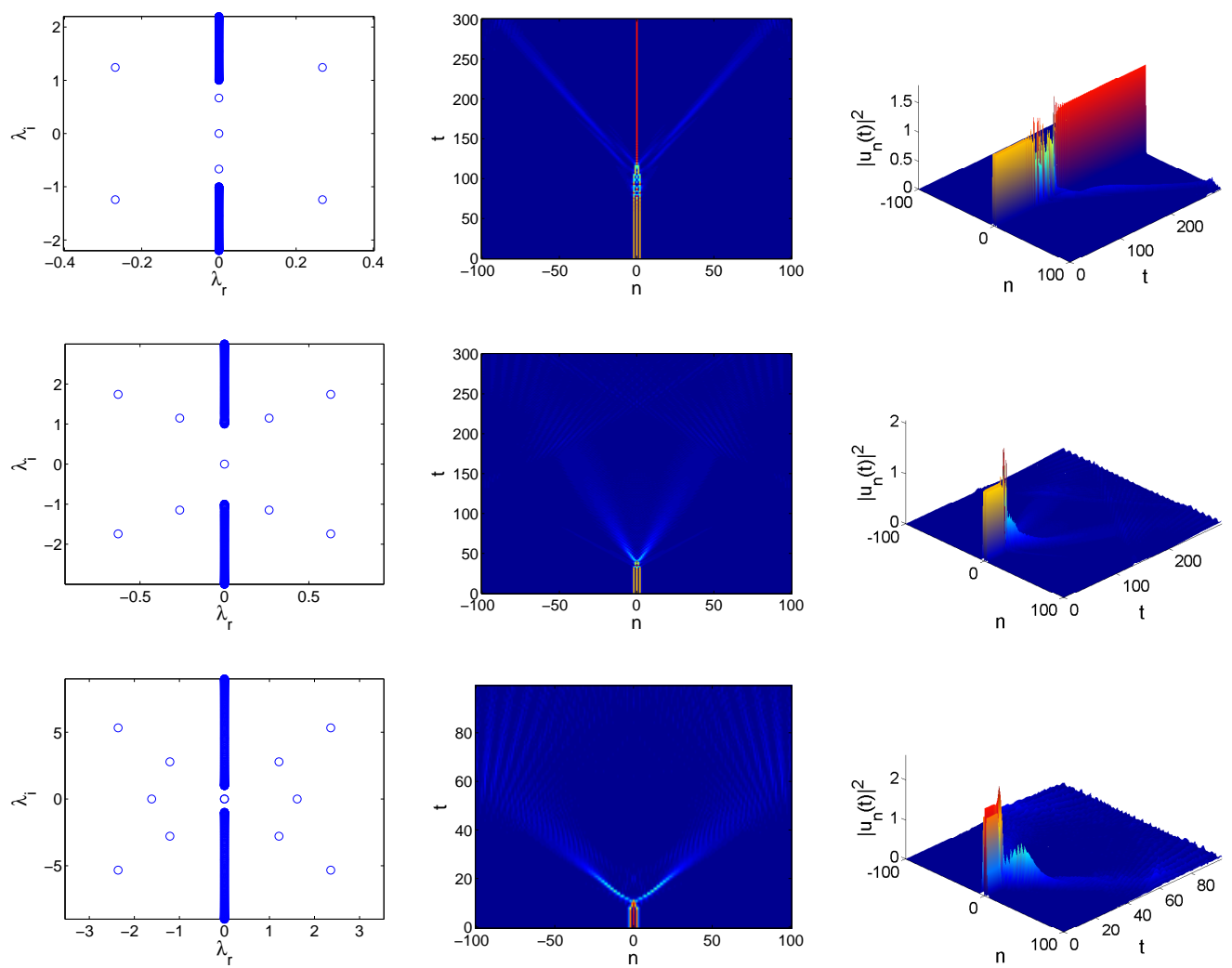

Fig. 10. The same as in Figs. 8 and 5, but for unstable three-humped solitons of type $(+1,0,-1,0,+1)$, with $\sigma=3$ and $\epsilon=0.3$ (top), $\epsilon=0.5$ (center), and $\epsilon=2$ (bottom).

bound states are unstable, while antisymmetric double solitons, and their signchanging triple counterparts, have finite stability regions, which were identified by means of theoretical prediction and numerical computation of the relevant linear stability eigenvalues. In addition, evolution of unstable solitons of various types has been explored with the help of direct simulations. It was found that they may self-trap into a persistent breather, or relax into a stable FS, or suffer complete decay.

The new results for the DNLS model with the quintic nonlinearity may be of relevance to the Tonks-Girardeau gas (the BEC of hard-core bosons) trapped in a strong optical lattice. For this and other applications, it should be quite interesting to extend the analysis to similar lattice models in higher-dimensions. Such investigations are currently in progress and will be reported in future publications.

Acknowledgements. PGK gratefully acknowledges support from NSF-CAREER, NSF-DMS-0505663 and NSF-DMS-0619492. JC acknowledges financial sup- 
port from the MECD project FIS2004-01183.

\section{References}

[1] P. G. Kevrekidis, K. Ø. Rasmussen, and A. R. Bishop, Int. J. Mod. Phys. B 15 (2001) 2833; T. Dauxois and M. Peyrard, Physics of Solitons (Cambridge University Press: Cambridge, 2005).

[2] D. N. Christodoulides and R. I. Joseph, Opt. Lett. 13 (1988) 794.

[3] H. S. Eisenberg, Y. Silberberg, R. Morandotti, A. R. Boyd, and J. S. Aitchison, Phys. Rev. Lett. 81 (1998) 3383.

[4] D. N. Christodoulides, F. Lederer, and Y. Silberberg, Nature 424 (2003) 817.

[5] J. W. Fleischer, G. Bartal, O. Cohen, T. Schwartz, O. Manela, B. Freedman, M. Segev, H. Buljan, and N. K. Efremidis, Opt. Exp. 13 (2005) 1780; F. Chen, M. Stepić, C. E. Ruter, D. Runde, D. Kip, V. Shandarov, O. Manela, and M. Segev, ibid. 13 (2005) 4314.

[6] V. O. Vinetskii and N. V. Kukhtarev, Sov. Phys. Solid State 16 (1975) 2414.

[7] M. Stepić, D. Kip, L. Hadžievski, and A. Maluckov, Phys. Rev E 69 (2004) 066618; L. Hadžievski, A. Maluckov, M. Stepić, and D. Kip, Phys. Rev. Lett. 93 (2004) 033901; A. Khare, K.Ø. Rasmussen, M. R. Samuelsen, and A. Saxena, J. Phys. A: Math. Gen. 38 (2005) 807; R. A. Vicencio and M. Johansson, Phys. Rev. E 73 (2006) 046602; T. R. O. Melvin, A. R. Champneys, P. G. Kevrekidis, and J. Cuevas, Phys. Rev. Lett. 97 (2006) 124101.

[8] A. Trombettoni and A. Smerzi, Phys. Rev. Lett. 86 (2001) 2353; G. L. Alfimov, P. G. Kevrekidis, V. V. Konotop, and M. Salerno, Phys. Rev. E 66 (2002) 046608; R. Carretero-González and K. Promislow, Phys. Rev. A 66 (2002) 033610 .

[9] F. S. Cataliotti, S. Burger, C. Fort, P. Maddaloni, F. Minardi, A. Trombettoni, A. Smerzi, and M. Inguscio, Science 293 (2001) 843; M. Greiner, O. Mandel, T. Esslinger, T. W. Hänsch, and I. Bloch, Nature 415 (2002) 39.

[10] M. A. Porter, R. Carretero-González, P. G. Kevrekidis, and B. A. Malomed, Chaos 15 (2005) 015115.

[11] R. S. MacKay and S. Aubry, Nonlinearity 7 (1994) 1623; S. Aubry, Physica 103D (1997) 201; S. Flach and C. R. Willis, Phys. Rep. 295 (1998) 181; G. P. Tsironis, Chaos 13 (2003) 657; D. K. Campbell, S. Flach, and Y. S. Kivshar, Phys. Today 57 (2004) 43.

[12] M. Sato, B. E. Hubbard, A. J. Sievers, B. Ilic, D. A. Czaplewski, and H. G. Craighead, Phys. Rev. Lett. 90 (2003) 044102; M. Sato and A. J. Sievers, Nature 432 (2004) 486. 
[13] R. Carretero-González, J. D. Talley, C. Chong, and B. A. Malomed, Physica D 216 (2006) 77.

[14] E. W. Laedke, K. H. Spatschek, and S. K. Turitsyn, Phys. Rev. Lett. 73 (1994) 1055.

[15] S. Flach, K. Kladko, and R. S. MacKay, Phys. Rev. Lett. 78 (1997) 1207.

[16] L. Bergé, Phys. Rep. 303 (1998) 259.

[17] L. Tonks, Phys. Rev. 50 (1936) 955; M. Girardeau, J. Math. Phys. (N.Y.) 1 (1960) 516.

[18] E. B. Kolomeisky, T. J. Newman, J. P. Straley, and X. Qi, Phys. Rev. Lett. 85 (2000) 1146.

[19] F. Kh. Abdullaev and M. Salerno, Phys. Rev. A 72 (2005) 033617; G. L. Alfimov, V. V. Konotop, and P. Pacciani ibid. A 75 (2007) 023624.

[20] B. A. Malomed and M. I. Weinstein. Phys. Lett. A 220 (1996) 91.

[21] C. Sulem and P.L. Sulem, The Nonlinear Schrödinger Equation, Springer-Verlag (New York, 1999).

[22] M. Johansson, S. Aubry, Yu.B. Gaididei, P.L. Christiansen, and K.Ø. Rasmussen, Physica D 119 (1998) 115.

[23] N. G. Vakhitov and A. A. Kolokolov, Izv. Vyssh. Uchebn. Zaved. Radiofiz. 16 (1973) 10120 [Radiophys. Quantum Electron. 16 (1973) 783].

[24] T. Kapitula, P.G. Kevrekidis, and B. Sandstede, Physica D 195 (2004) 263; see also ibid 201 (2005) 199.

[25] T. Bountis, H. W. Capel, M. Kollmann, J. C. Ross, J. M. Bergamin, and J. P. van der Weele, Phys. Lett. A 268 (2000) 50.

[26] T. Kapitula, P. G. Kevrekidis, and B. A. Malomed, Phys. Rev. E 63 (2001) 036604.

[27] V. Koukouloyannis and S. Ichtiaroglou, Phys. Rev. E 66 (2002) 066602; V. Koukouloyannis, Phys. Rev. E 69 (2004) 046613.

[28] G. Kalosakas, Physica D 216, 44 (2006).

[29] B. A. Malomed, Progr. Opt. 43 (2002) 71.

[30] D. E. Pelinovsky, P. G. Kevrekidis, and D. J. Frantzeskakis, Physica D 212 (2005) 1.

[31] G. L. Alfimov, V. A. Brazhnyi, and V. V. Konotop, Physica D 194 (2004) 127. 